\title{
Construction of Virtual Learning Environments of Lushan Mountain
}

\author{
Weijun Yang ${ }^{1}$, Jianhua Gong ${ }^{1}$, Lihui Zhang ${ }^{1}$, and Hongli Fan ${ }^{2}$ \\ ${ }^{1}$ Institute of Remote Sensing Applications, Chinese Academy of Sciences, \\ P.O.Box9718, Beijing, 100101, P.R. China, \\ wjy172@163.com \\ ${ }^{2}$ Institute of Automotive Industry \\ ShiYan, HuBei, 442002, P.R. China
}

\begin{abstract}
Organizing a fieldwork in LuShan Mountain consumes much money and time, but the result is not effective. This demands a virtual learning environment of LuShan Moutain. Some methods of data collection based on mobile equipments are adopted. The data collection system constructs a collaborative 3D modeling environment, in which students can do goecollaboration. In this paper, some technologies to support data collection are discussed.
\end{abstract}

Keywords: Virtual Learning Environment, Virtual Geographic Environment, Virtual Geographic Fieldwork, Data Collection, Collaborative 3D Modeling.

\section{Introduction}

A virtual geographic environment (VGE) is a multi-user shared, intelligent, and virtual environment representing the real geographic environment to conduct geo-spatial analysis, to carry out geo-visualization, to support collaborative work, planning and decision making, as well as to serve training, geographic education, and entertainment [2]. VGE can represent the natural world and enables a person to explore and interact with objects in cyberspace.

In this paper, some technologies such as collaborative $3 \mathrm{D}$ modeling between multi PCs or mobile equipments are discussed.

\section{Virtual Geographic Fieldwork}

Virtual learning environment uses the combination of long-distance communication tools and multimedia technologies such as VR to provide an online learning path for students. Virtual learning environment (VLE), not only provides rich teaching patterns and teaching contents, but also helps improve learners' ability of analyzing problems and exploring new concepts [5]. VR is the most feasible technology for us to create VLE. Virtual geographic fieldwork is a key application of VGE in the field of geographic fieldwork. It also forms a virtual learning environment of Lushan Mountain. 
Lushan Mountain, situated in the north of Jiangxi Province, at $115^{\circ} 52^{\prime}$ $116^{\circ} 08 \mathrm{E}^{\prime} 29^{\circ} 26-29^{\circ} 41^{\prime} \mathrm{N}$, occupies an area of 302 square kilometers. Lushan Mountain is famous for its beautiful natural landscape. Not just so, because of its special terrain, geology, etc, it has become a perfect fieldwork place for students of many majors, such as GIS, Geology, Remote Sensing, etc. Why a virtual geographic fieldwork is necessary?

First, organizing such a fieldwork consumes so much money and time. And students often complain that the result is not effective. The data collection became a mess because of no trim and no accumulation. It's like a travel more than a fieldwork. Second, the data collection will certainly do some destroy to the environment of Lushan Mountain. This is also a problem in the outdoor fieldwork. Third, the information stored in the computer can be permanent, but specimens, such as plants, cannot last a long time.

These all call for a virtual fieldwork system, students can explore in the virtual geographic environment and acquire knowledge in a virtual 3D scene. Above disadvantages can be avoided. So, at the first, we need a data collection system, which can be applied to the data collection of virtual fieldwork. It forms an environment in which many modelers can do collaborative 3D modeling.

\section{Data Classification and Structure}

The data of outdoor fieldwork can be classified to six categories, such as plant, rock, soil, terrain, geology and artificial object.

Dem and image objects are the information of the terrain. Point, linear, surface, body objects are base types of CyberCity GIS. Point object is implemented to afford the ability to integrate with CAD models. The point object can describe such features as trees, annotations, static CAD models. Trees are represented by transparent textures [3]. Some artificial objects can be represented by CAD models of point object and inserted to the scene.

The virtual geographic fieldwork is required to afford the ability of spatial analysis. So the data structure is very important. Similar to the formal data structure (FDS) [1] [4] [6], the CyberCity models are grouped into six different object types: DEM object, image object, point object, linear object, surface object, and body object. 3D point object, the cube object, has the simplest spatial extension (the 3D position, the azimuth and the box size) and sometimes special attributes such as CAD models in 3DS format.

\section{Offline Data Collection Though PDA}

The first method is offline data collection though PDA. Students can collect the text and picture information though PDA. The position information is acquired by GPS. After coming back, they transport the data to the PC and build the 3D models. The data collection and modeling are separate. So an environment of offline data collection is constructed. 
In the process of data collection, a linkage file is created. In this file, the relationship of the information such as pictures, texts, videos and position is recorded. After the students come back, they can add the data to the database in a collaborative 3D modeling environment. Some support technologies must be adopted to support it. The most important one is to integrate some theories of $3 \mathrm{D}$ interactive visualization to the collaborative $3 \mathrm{D}$ modeling environment, such as dynamic loading by data page, view frustum culling, etc.

\section{Online Data Collection Though Mobile Phone}

The second method is online data collection though mobile phone. The students collect the information though mobile phone and GPS. After some simple data handling in mobile phone, they can send the data to the database server directly after simple handling. At the same time, the 3D scene is built. After data collection, the 3D data is created at the same time. So an environment of online data collection is constructed.

J2ME is Sun Microsystems' answer to a consumer wireless device platform. J2ME allows developers to use Java language and the J2ME wireless toolkit to create applications and programs for wireless and mobile devices. The Connected Limited Device Configuration (CLDC) is the J2ME configuration for smaller handheld devices that are usually battery operated and low in memory with limited processing power and low bandwidth. CLDC defines the base set of application programming interfaces and a virtual machine for resource-constrained devices like mobile phones, pagers, and mainstream personal digital assistants. When coupled with a profile such as the Mobile Information Device Profile (MIDP), it provides a solid Java platform for developing applications to run on devices with limited memory, processing power, and graphical capabilities.

In MIDP, there are two methods to communicate between MIDlet and Servlet. The first method is using Java Server Pages as server component. The second method is communicating with Servlet directly. Java Server Pages are mainly applied in the representation layer of WEB applications. Servlet is more adapted to handle logic or communicate with client as a control. HTTP protocol is adopted to visit web data and service.

\section{Collaborative 3D Modeling}

\subsection{Collaborative Modeling Between PCs}

Integrating interactive visualization to $3 \mathrm{D}$ modeling is a revolutionary change to traditional 3D modeling method based on subareas. It forms an environment in which many modelers can carry collaborative work. Some technologies, such as network, 3D interactive visualization, etc, are used to establish this environment. Many modelers can create 3D models in a large scene with great amount of data. Thus, a collaborative 3D modeling environment is constructed to support 3D modeling for 3DGIS, CyberCity GIS, virtual reality, VGE, 3D game, etc. 


\subsection{Collaborative Modeling Between Mobiles}

Because there is no 3D graphic interface on mobile equipments, the theories of collaborative modeling between PCs are not used on them. But if there is a demand of graphic interface on mobile equipments in the future, the theories are also useful.

\subsection{Collaboration Between Mobiles and PCs}

In some applications, the PCs and mobiles may be both needed. So the collaboration between PCs and mobile equipments must be considered. Four communication patterns are put forward. These are S2S (Static to Static), M2S (Mobile to Static), S2M (Static to Mobile), and M2M(Mobile to Mobile). S2S means the communication between the persons using different computers in the rooms. M2S means the outdoor person with mobile equipment communicates with the person using computer in the room. S2M means the person using computer in the room communicates with the outdoor person with mobile equipment. M2M means the communication between the outdoor persons with mobile equipments. In the collaborative modeling between mobiles, only the pattern M2S is implemented. But in other applications, some other patterns may be used.

\section{Acknowledgements}

This research is partially supported by the National High-tech R\&D Program (863 Program) No. 2006AA12Z204 and the Knowledge Innovation Program of the Chinese Academy of Sciences, Grant No. Kzcx2-yw-126-01.

\section{References}

1. Gong, J., Li, D.: Object-oriented and Integrated Spatial Data Model for Managing Image, DEM, and Vector Data, Photogrammetric Engineering \& Remote Sensing (2000), 66(5):619-623.

2. Gong, J., Lin, H.: A geographical perspective on online virtual reality (2001), pp.62-63.

3. Jurgen, D.: An object-oriented approach for integrating 3D visualization systems and GIS, Computers \& Geosciences, 26 (2000) 67-76

4. 4.Molenaar, M.: A topology for 3D vector maps, ITC Journal (1992), (1): 25-33.

5. Pan, Z., Cheok, A., Yang, H.: Virtual reality and mixed reality for virtual learning environments. Computers \& Graphics (2005)

6. Wang, X.: A Hybrid GIS for 3-D City Models, In: International Archives of Photogrammetry and Remote Sensing (2000). Vol. XXXIII, Part B4, pp. 1165-1172. 